\title{
Sputtering of Neutral Molecules and Molecular lons from the Adenine Crystal Surface Induced by the UV Picosecond Laser Pulse
}

\author{
V.S. ANTONOV, V.S. LETOKHOV, YU.A. MATVEYETS and A.N. SHIBANOV \\ Institute of Spectroscopy, USSR Academy of Sciences, SU-142092, Moscow \\ Region, Troitsk, USSR
}

This paper presents the results of observation of sputtering of neutral molecules and ions from the crystal adenine surface induced by fourth-harmonic Nd:YAG laser radiation with a pulse duration of $30 \mathrm{ps}$. The energy fluence of laser pulses was in the region (1-3) $\times 10^{-4}$ $\mathrm{J} / \mathrm{cm}^{2}$. The kinetic energy distribution of the sputtered molecules spreads up to $0.7 \mathrm{eV}$. The experiment shows that the threshold of adenine molecular ion sputtering is connected with absorbed energy density in upper layers of the crystal surface but not by laser radiation intensity.

\section{INTRODUCTION}

Previously ${ }^{1,2}$ we reported on observation of molecular ion sputtering of anthracene and DNA bases from a crystal surface induced by nanosecond pulsed UV laser radiation. This radiation wavelength coincides with the electron absorption bands of these crystals. The main feature of the observed phenomenon is its nonthermal nature: heating by laser radiation of a crystal surface under experimental conditions did not exceed $20-50^{\circ} \mathrm{C}$. More detailed experiments ${ }^{2}$ with adenine crystals and nanosecond pulses 
of the $\operatorname{KrF}$ laser $(\lambda=249 \mathrm{~nm})$ revealed a strong dependence $\left(F^{6}\right)$ of molecular ion yield on the energy fluence $F$ of laser radiation, and high kinetic energy of molecular ions up to $3 \mathrm{eV}$. This shows the complex nature of processes resulting in ions sputtering from the crystal surface. Some mechanisms were proposed ${ }^{2}$ to explain ion sputtering but their roles in the process were not appreciated for lack of experimental data.

There are two important questions concerning the observed effect: (a) whether the sputtering threshold is connected with the intensity of laser radiation or its energy fluence; (b) is the neutral molecule sputtering taking place parallel with ion sputtering? This paper presents the results of ion and neutral molecule sputtering from the adenine crystal surface irradiated by picosecond UV laser pulses $(\tau=30 \mathrm{ps})$. These results have been compared with experiments ${ }^{1,2}$ where nanosecond pulses were used. Ions sputtered from the crystal surface were detected by a time-of-flight mass spectrometer. Neutral molecules sputtered from the crystal surface were detected by photoionization by a $\mathrm{KrF}$ laser in the volume above the surface.

\section{Experimental setup}

Our previous work ${ }^{2}$ describes an experimental setup for the investigation of laser-induced sputtering of ions. A finely dispersed crystalline powder (Zelstoffabrik, Waldhof) was placed on the repellent electrode of the timeof-flight mass spectrometer ion source. A single picosecond pulse of fourthharmonic Nd:YAG laser radiation of $266 \mathrm{~nm}$ wavelength was directed at the sample on the $10^{\circ}$ angle to the plane of the repellent electrode (Figure 1). The sputtered ions repelled from the ion source region by the electric pulse of $125 \mathrm{~V} / \mathrm{cm}$ and after mass separation in the time-of-flight mass analyzer were recorded with an electron multiplier. The signal from the multiplier was recorded by a memory oscilloscope.

To detect the sputtered neutral molecules the time-delayed $\mathrm{KrF}$ laser pulse was used to photoionize sputtered molecules above the surface. Molecular photoionization was effected according to the two-step scheme ${ }^{3}$ through an intermediate electron-excited state, because the $\mathrm{KrF}$ laser photon energy equals $5 \mathrm{eV}$ and the ionization potential of the adenine molecule is $8.3 \mathrm{eV} .{ }^{4}$ The $\mathrm{KrF}$ laser pulses were focused by a quartz lens $f=0.4$ $\mathrm{m}$, the focus region was at a distance of $4 \mathrm{~mm}$ above the sample. The energy fluence of the pulses in the focus region was $0.5-1 \mathrm{~J} / \mathrm{cm}^{2}$. The $\mathrm{KrF}$ laser pulse delay time with respect to the UV picosecond laser pulses irradiating the adenine crystal surface was varied in the region (2-10) $\mu \mathrm{s}$. 

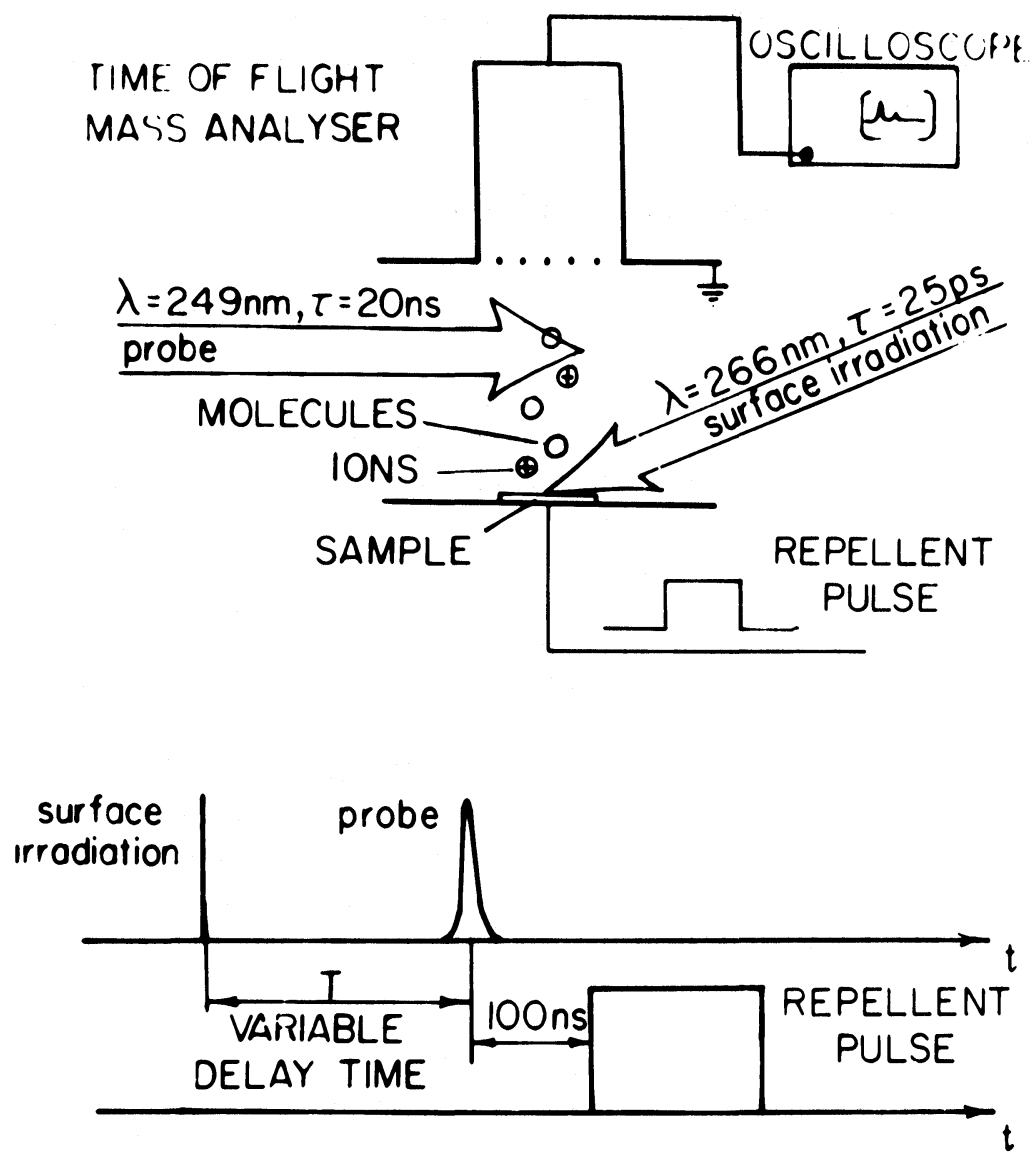

FIGURE 1. Scheme of the experiment. The lower portion is a time diagram of laser pulses and of the repellent electric pulse.

This allowed measurement of the velocity distribution of sputtered molecules in the region $(4-20) \times 10^{4} \mathrm{~cm} / \mathrm{s}$.

The time-of-flight mass spectrometer had a resolution of 200 at a halflevel amplitude. The mass identification accuracy for the ions sputtered from the crystal surface equalled plus or minus 1 a.u. For the molecules ionized by $\mathrm{KrF}$ laser above the surface the mass identification accuracy was plus or minus 7 a.u. This is connected with the nonoptimal working regime of the mass spectrometer in case of simultaneous detection of ions 
sputtered from the surface and ions formed as a result of photoionization of molecules above the surface. The residual gas pressure in the mass spectrometer was $10^{-7}$ Torr. Oil-free pumping was used for evacuation.

\section{Experimental results and discussion}

Investigation of mass spectra of ions resulting from picosecond pulse ( $\tau$ $=30 \mathrm{ps}, \lambda=266 \mathrm{~nm}$ ) irradiation of adenine crystals shows that as in the case of Ref. 2, where $\mathrm{KrF}$ laser pulses $(\tau=20 \mathrm{~ns}, \lambda=249 \mathrm{~nm})$ were used, the dominant component is a molecular ion. The sputtered ion yield, as in Ref. 2, had a strong dependence on the laser pulse energy fluence. Exact measurements of the sputtered ion yield dependence on the picosecond pulse energy fluence was difficult for the fluctuations of spatial energy distribution in the laser beam. The important difference is the lower threshold of $1.5 \times 10^{-4} \mathrm{~J} / \mathrm{cm}^{2}$ for the ion sputtering with respect to the threshold of $8 \times 10^{-4} \mathrm{~J} / \mathrm{cm}^{2}$ in the case of $\lambda=249 \mathrm{~nm}, \tau=20 \mathrm{~ns}$. But in the last case the absorption coefficient of the adenine crystal for the wavelength of $249 \mathrm{~nm}\left(\mathrm{~K}_{249}=3.8 \times 10^{4} \mathrm{~cm}^{-1}\right)$ is four times less than that for $\lambda=266 \mathrm{~nm}\left(\mathrm{~K}_{266}=1.6 \times 10^{5} \mathrm{~cm}^{-1}\right){ }^{5}$ Taking into account this fact, one can conclude that the threshold of absorbed energy density for the molecular ion sputtering is the same for both cases, though the intensity of the picosecond laser pulse was of two orders of magnitude higher than that of the nanosecond one. It follows from the analysis of time duration of the molecular ion signal that as in Ref. 2 the kinetic energy distribution of the sputtered ions spreads up to several $\mathrm{eV}$.

Figure 2 shows the multiplier signal corresponding to the mass spectrum region near the adenine molecular ion. The oscillogram $\mathrm{A}$ was obtained when the sample crystals had been irradiated by the picosecond pulse of the fourth-harmonic Nd:YAG laser with energy fluence $2.5 \times 10^{-4} \mathrm{~J} / \mathrm{cm}^{2}$. The signal amplitude is equal to $10^{2}$ ions per pulse. The B oscillogram was obtained when only $\mathrm{KrF}$ laser pulses focused above the surface were used. The small signal in this case is related to the photoionization of organic molecular background and adenine vapor. The $C$ oscillogram was obtained when both laser pulses with delay time between them $\Delta t=4 \mu \mathrm{s}$ were used. At the same time as the pulse corresponding to adenine molecular ions sputtered from the surface, an additional pulse can be seen corresponding to the photoions obtained from the neutral molecules above the surface. This last pulse depends on the $\mathrm{KrF}$ laser energy fluence as $F^{1.5}$. The deviation from the square law dependence apparently is related 


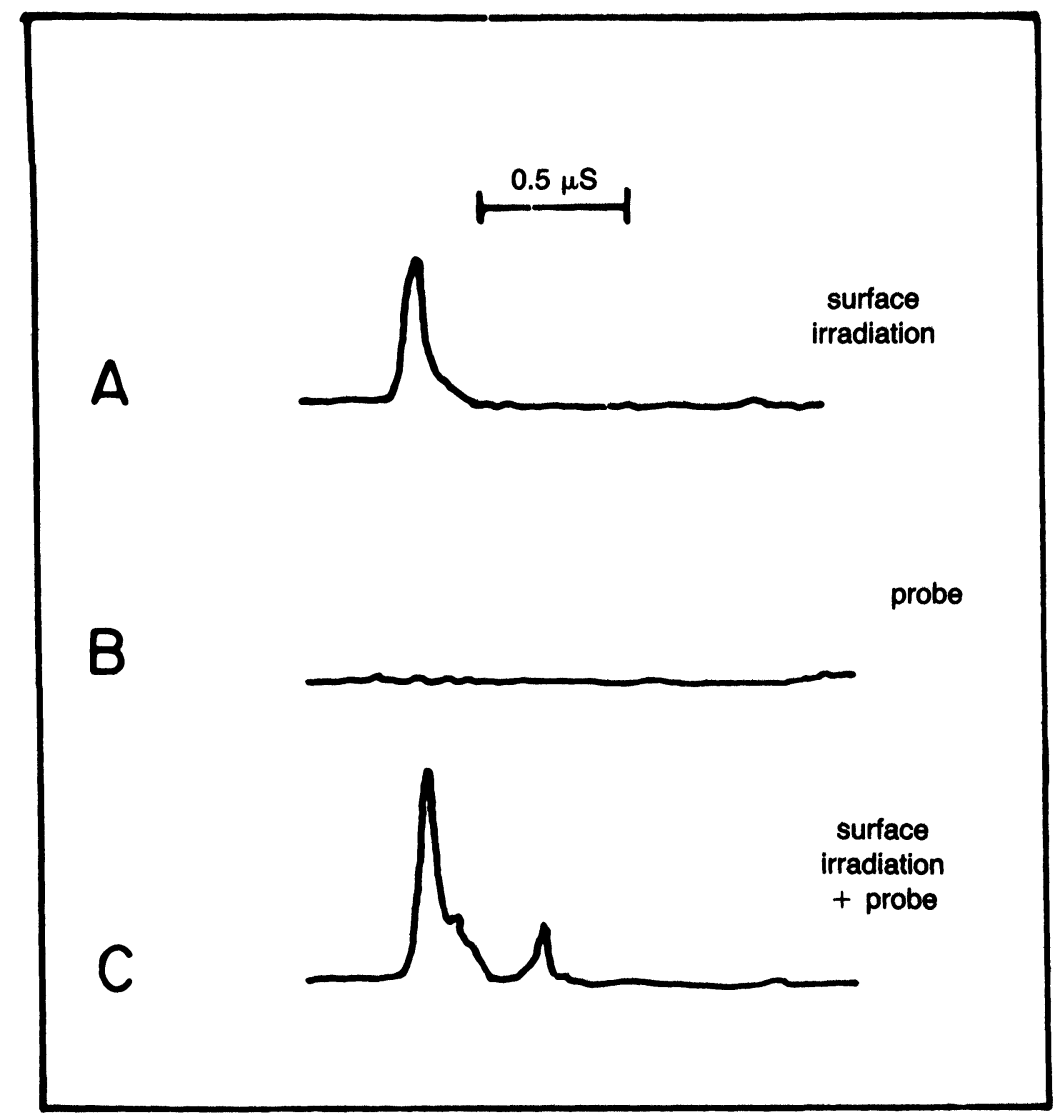

FIGURE 2. Oscillograms of electron multiplier signals. (A) the crystal surface being irradiated, $\lambda=266 \mathrm{~nm}, \tau=30 \mathrm{ps}$. (B) the $\mathrm{KrF}$ laser pulses probing above the surface, $\lambda$ $=249 \mathrm{~nm}, \tau=20 \mathrm{~ns}$. (C) both pulses used with delay time of $4 \mu \mathrm{s}$.

to the saturation of the two-step photoionization yield at the energy fluence $F=0.5 \mathrm{~J} / \mathrm{cm}^{2}$. Based on the ions, time-of-flight estimations of sputtered neutral molecular mass give $m=(140 \pm 7)$ a.u., which equals the adenine molecular mass of 135 a.u. within the range of experimental error. Taking into account the less efficient detection of neutral molecules, one can conclude from Figure $2 \mathrm{C}$ that the number of sputtered adenine molecular ions is approximately equal to the sputtered molecules. But when the energy of the irradiating laser pulses decreases the number of sputtered molecules 
decreases more slowly than that of ions. This leads to the predominance of the neutral molecule sputtering process. As laser energy is increased the ion sputtering process becomes dominant.

Figure 3 shows the velocity distribution of the sputtered molecules in the region (4-20) $\times 10^{4} \mathrm{~cm} / \mathrm{s}$. This distribution was measured by variation of delay time of $\mathrm{KrF}$ laser pulses time-delayed with respect to the picosecond pulses irradiating the surface in the region of $(2-10) \mu s$. Calculating the velocity, it was suggested that after the irradiation of the crystal surface the sputtering process had been proceeding less than $1 \mu \mathrm{s}$. Figure 3 shows that maximum velocities of the sputtered molecules were up to $10^{5} \mathrm{~cm} / \mathrm{s}$, i.e., corresponding to $0.7 \mathrm{eV}$. The dotted line represents the Maxwell velocity distribution of molecules with $t=1250^{\circ} \mathrm{C}$. But according to the estimation, the heating of the crystal surface under experimental conditions does not exceed $30^{\circ} \mathrm{C}$. This estimation suggests that the absorbed laser

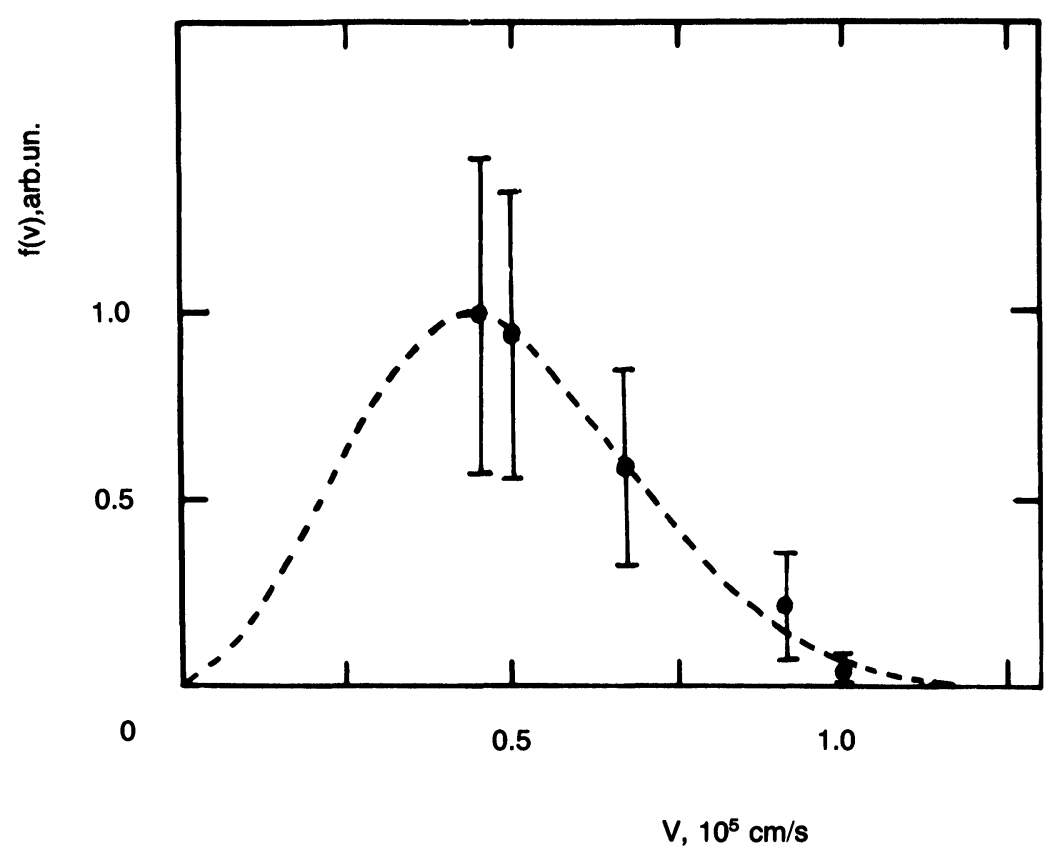

FIGURE 3. Velocity distribution of the sputtered neutral molecules. The dotted line represents the Maxwell distribution with $t=1250^{\circ} \mathrm{C}$. 
energy is thermolized in the upper layers of the adenine crystals. This fact points out the nonequilibrium nature of molecular sputtering.

\section{CONCLUSIONS}

The experimental results have shown that for the case of picosecond irradiation $(\lambda=266 \mathrm{~nm})$ of the adenine crystal the energy fluence threshold of molecular ions is five times lower than that of experimental conditions in work where the nanosecond $\mathrm{KrF}$ laser pulses $(\lambda=249 \mathrm{~nm})$ were used. ${ }^{2}$ But taking into consideration the difference of absorption coefficients for the radiation with $\lambda=249 \mathrm{~nm}$ and $\lambda=266 \mathrm{~nm}$, the absorbed energy density threshold was the same in both cases.

Near the threshold of ion sputtering the sputtering of neutral molecules with mass $m=140 \pm 7$ a.u. (near the adenine mass $m=135$ a.u.) was observed. When the energy fluence of picosecond laser pulses was in the region $1 \pm 2 \times 10^{-4} \mathrm{~J} / \mathrm{cm}^{2}$ the number of sputtered neutral molecules exceeded the number of ions, but with an increase of pulse energy fluence ion sputtering became dominant.

\section{References}

1. V. S. Antonov, V. S. Letokhov and A. N. Shibanov, Pis'ma Zh. Exp. Teor. Fiz. 31, 471 (1980).

2. V. S. Antonov, V. S. Letokhov and A. N. Shibanov, Appl. Phys. 25, 71 (1981).

3. V. S. Antonov and V. S. Letokhov, Appl. Phys., to be published.

4. B. I. Verkin, L. F. Sukhodub and I. Ya. Yanson, Dokl. Akad. Nauk. SSSR Ser. Khim. 228, 1452 (1976).

5. K. A. Cherkasov, M. N. Kiselyova and N. Ya. Dodonova, Opt. Spektrosk. 45, 1126 (1978). 